\title{
A Heterogeneous Dependency Graph as Intermediate Representation for Instruction Set Customization
}

\author{
Kang Zhao Jinian Bian Sheqin Dong ${ }^{1}$ \\ EDA Lab, Dept. of Computer Science \& Technology \\ Tsinghua Univ., Beijing 100084, China, +86-10-62785564
}

\begin{abstract}
A heterogeneous dependency graph (HDG) defined as the intermediate representation for instruction set automated customization is presented in this paper. The main motivation of this model is to constructs a unified internal specification that bridges the gap from the application benchmarks to the instruction set customization. To represent the necessary information required by the instruction set customization process, basic elements and heterogeneous structures including pipelining, parallel, branch and loop are presented in detail.
\end{abstract}

Keywords: Heterogeneous dependency graph, HDG, design automation, instruction set customization, ASIP.

\section{Introduction}

Application Specific Instruction-set Processor (ASIP) is a processor designed for a set of particular applications, which provides a good tradeoff between efficiency and flexibility [1]. To face the challenges of high efficiency and time-to-market pressure, automatic customization of ASIP to the application requirements has become an attractive technology [2].

In ASIP synthesis, instruction set customization plays a kernel role, which consists of the generation of whole instruction set and corresponding assembly code. In order to find an efficient solution for the instruction set customization, designers must bridge the gap with an intermediate representation which can satisfy the requirements of the customization process.

There are many candidate representations which have been widely used. Control and Data Flow Graph (CDFG) has been widely used in high level synthesis [3] [4]. As an intermediate representation, CDFG performs the conversion of the behavior description into a single graph used by the scheduling and allocation algorithm in synthesis. CDFG Toolkit [5] is even produced by Design Automation Lab in Seoul
National University. Furthermore, a powerful three layered CDFG including DFG, CFG and Hierarchical Task Graphs have been presented in SPARK [6], which resolves the existing problem that abstraction level is too low for the range of coarse-grain and finegrain parallelizing compiler transformations.

However, there are two intrinsic limitations in the specifications mentioned above. First, main structures in those models are sequential, which cannot satisfy the parallel optimizations in the instruction set customization. Second, those models focus on certain application problems and their specific definitions are not suitable for instruction set customization.

To address these issues, we present a novel model named as Heterogeneous Dependency Graph (HDG), which consists of four heterogeneous structures, pipelining, parallel, branch and loop. As the unified intermediate representation, HDG satisfies the basic requirements of three sub-processes of instruction set customization, basic instruction set selection, complex instruction generation and assembly code generation.

The rest of the paper is organized as follows. In Section 2, the instruction set customization framework and corresponding requirements for the intermediate representation are presented. Then the basic elements of HDG are defined in Section 3. Section 4 presents the four main heterogeneous structures in detail. To understand it deeply, an experimental example for HDG is presented in Section 5. Finally, an analysis compared with CDFG is done to support our proposed model HDG. The last section draws the conclusion.

\section{Application Requirements}

Instruction set customization is a process which gradually moves the design to lower levels, as shown in Figure 1. It starts with the behavior specification of the application benchmarks written with high level languages. During instruction set customization, basic instructions are selected from a pre-designed library and complex instructions are generated by profiling

\footnotetext{
${ }^{1}$ Work supported in part by National Natural Science Foundation of China under grant NSFC-90207017, and NSFC-60236020; National Basic Research Program of China (973) under grand 2005CB321605.
} 


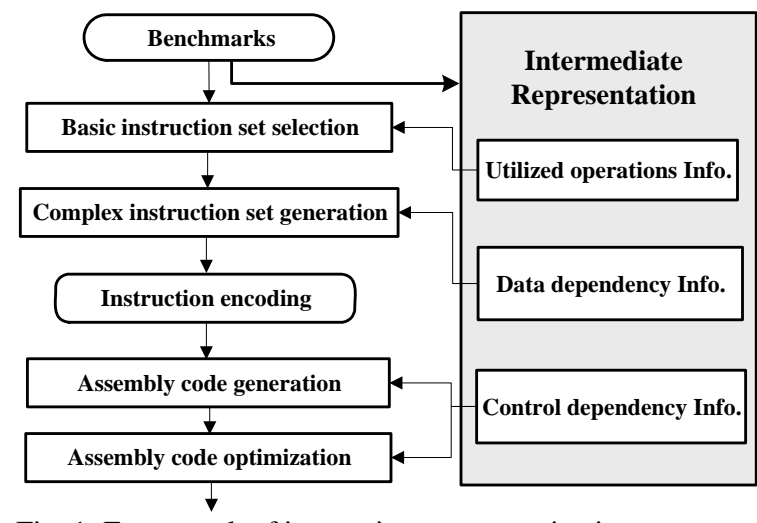

Fig. 1: Framework of instruction set customization.

data dependency information in the benchmark. After instruction encoding, the assembly code is generated and optimized to improve the processor performances.

From this framework we can find that the gap between the application benchmarks and instruction set customization process must be bridged by using an intermediate representation. There are three essential requirements this representation should satisfy:

(1) This representation should be able to extract and represent the information of the utilized atomic operations from benchmarks. As explained previously, the motivation of instruction set selection is to match the atomic operations to the basic instructions in the library, so during the conversion from the benchmark written in high level language to the intermediate representation, the utilized operations must be represented with a proper format.

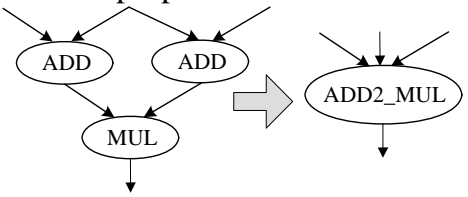

Fig. 2: An example of complex instruction generation.

(2) The intermediate representation must be able to supply the data dependency information for the complex instruction generation. Furthermore, it also has the hierarchical feature. As shown in Figure 2, the complex instruction is generated by combining atom instructions, which should have same numbers of input and output. So the intermediate representation must represent the dependencies from fine to coarse granularity via the hierarchical feature. The essential problem of the complex instruction generation is to determine the subset of an application's data flow graph, as shown in Figure 3, which can improve the performances of the final processor. For example, we combine the atomic operations on the critical path of the graph and implement with one instruction, the executable rate of the processor must be improved. Therefore, to find an efficient solution for instruction set customization, those data dependency information is very necessary in the intermediate representation.

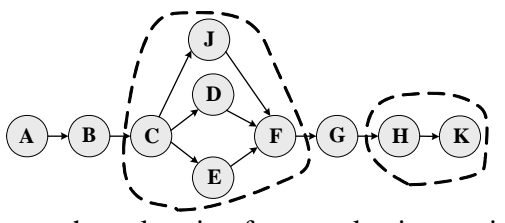

Fig. 3: Sub-graph exploration for complex instructions.

(3) Control dependency information must be contained in the intermediate representation for the assembly code generation and optimization. After the instruction set is generated, the benchmark should be converted to assembly language to execute on the processor, so basic structures in the benchmark such as branch and loop must be represented in intermediate representation. Furthermore, to increase the execution rate optimization on the assembly code will be utilized, such as loop unrolling, parallelism and pipelining. Therefore, the intermediate representation should also be able to represent those heterogeneous structures.

From the analysis of the application requirements above, we present a novel model as the intermediate representation which named as HDG. In the following sections we will give the detailed definitions of HDG.

\section{Basic Elements of HDG}

As the unified representation for the instruction set customization, HDG consists of two basic elements, node and flow. It is defined as flows:

$$
\begin{aligned}
& \text { HDG }=<V, E> \\
& V=\left\{v_{1}, v_{2}, \ldots v_{m}\right\} \\
& E=\left\{e_{1}, e_{2}, \ldots e_{n}\right\}
\end{aligned}
$$

Where $V$ is the set of nodes, which represent the utilized operations, states, variables, or tasks. $E$ is the set of flows, which represent a data flow or a control token's transmission. And to represent the hierarchical feature, we add an option to the parameters in $V$ :

$v=<i d$, type, in_flows, out_flows, father, child $>$

where father is a pointer which links to the hierarchical node $v$ belongs to, and child points to the set of nodes which are captured in $v$.

$$
\begin{aligned}
& V=O N \cup T N \cup C N \\
& C N=S N \cup M N \cup F N \cup J N \cup C L N \cup E N \\
& E=C F \cup D F
\end{aligned}
$$

The nodes in HDG consist of three types: operation node $(O N)$, transport node $(T N)$ and control node $(C N)$. $O N$ stands for one operation or task, $T N$ represents an event which results in a transport of data, and $C N$ is the symbol of special structures. In addition, $C N$ consists of select node $(S N)$, merge node $(M N)$, fork node $(F N)$, join node $(J N)$, clear node $(C L N)$ and encase node $(E N)$. The representations of these control nodes are shown in Figure 4. Furthermore, $C F$ and $D F$ represent the control flow and data flow respectively.

The difference of $S N$ and $F N$ is that $S N$ sends the control token to only one output flow and $F N$ sends 
the same token to each output flow. Similarly, $M N$ is fired only if one token from input flows arrives, but $J N$ waits until all the tokens from input flows arrive. CLN and $E N$ are mainly used in pipelining structures.

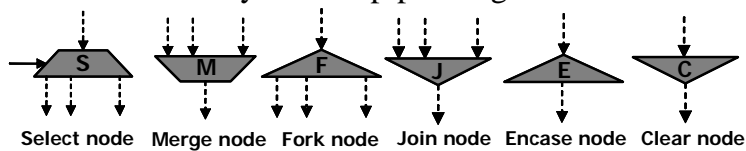

Fig. 4: Graph representation for control nodes.

\section{Heterogeneous Structures}

In order to represent the features for the instruction set customization, special structures in HDG are presented, including pipelining and parallel supporting assembly code optimization, and branch and loop supporting basic structures in the benchmark.

\subsection{Pipelining}

Pipelining is a special structure of parallel execution which is widely used to represent the characters of hardware. Recently effective hardware generation to deal with complex loops requires the ability to exploit the pipelining. Furthermore, pipelining also has an important effect on power dissipation, which has been validated by Actel Corporation [7].



(a)

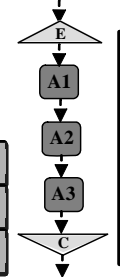

(b)

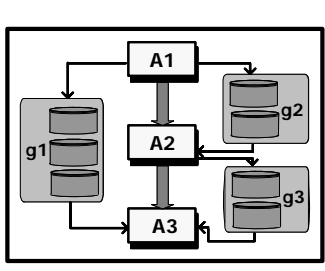

(c)
Fig. 5: (a) An example of pipelining; (b) Pipelining in HDG; (c) Data storage when pipelining.

Therefore, for the sake of widely application of pipelining in the whole design, the intermediate model HDG must be able to represent this structure. A typical example for pipelining is shown in Figure 5(a). There are three operations $A 1, A 2$ and $A 3$ which forms a three-stage pipelining, including encase stage, core stage and clear stage. In HDG pipelining is composed of encase and clear nodes, as illustrated in Figure 5(b).

HDG also supports the specification of the data storage when pipelining. Dummy registers in $T N s$ are defined to fulfill this task. Between the pipeline stages, additional dummy registers are added into $T N$ to store variables in different stages. As the example shown in Figure 5(c), variable $g 1$ stores data from operation $A 1$ to $A 3$, whereas $g 2$ and $g 3$ from operation $A 1$ to $A 2$, and $A 2$ to $A 3$ respectively. Three dummy registers in $g 1$ and two dummy registers in $g 2$ and $g 3$ ensure that $g 1$ transfer data in the second pipelining period from $A 1$ to $A 2$ and in the third pipelining period from $A 1$ to $A 3$.

\subsection{Parallel}

Parallel is a simple but widely used structure, which represents the feature of hardware design very well. Many input languages support this structure, such as VHDL, Verilog, SpecC, SystemC. Furthermore, in the hardware design level, parallel computation and data transmission is connatural.

Parallel structures are represented mainly with fork and join nodes in HDG. An example is shown in Figure 6(a), where A1, $A 2$ and $A 3$ run in parallel. They all start simultaneously when the fork node is started up. Once all of them have completed their execution, the token will be route to the join node.

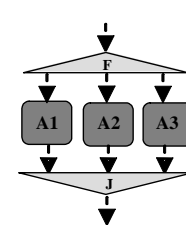

(a)





(c)

(d)
Fig. 6: (a) Parallel; (b) Branch; (c) while loop structure; (d) do-while loop structure.

\subsection{Branch}

Branch is one of the most widely used structures. A majority of input languages have this structure, in spite of software languages and hardware languages such as $\mathrm{C}$ and VHDL.

In HDG, branch structure begins with a select node and ends with the corresponding merge node, as illustrated by Figure 6(b). In this definition, the condition estimation part is not contained in the branch structure; instead, it only communicates with the branch by the data input flow of the select node, which controls the directions in the branch.

\subsection{Loop}

Loop structure is also a familiar structure in high level languages, which contain while and do-while two types. The graph representation for loop is shown in Figure 6(c) (d). It begins with a merge node and ends with the corresponding select node. The difference between while type and do-while type is that the positions of condition estimation node and loop body are just opposite.



Fig. 7: A section of MPEG as an example. 


\section{An Experimental Example}

To understand the novel model deeply, we choose one section of MPEG as an example, as shown in Figure 7. Because the computation for the motion picture is very large, many optimized process should be done. Several guidelines should be followed when turning MPEG to HDG: (1) All possible parallelism operations should be exposed explicitly using the concurrent-execution constructs in HDG, such as parallel and pipelining. (2) Hierarchical nodes should be chosen with appropriate size and utilized to group related leaf operations, which are the smallest indivisible units in HDG.

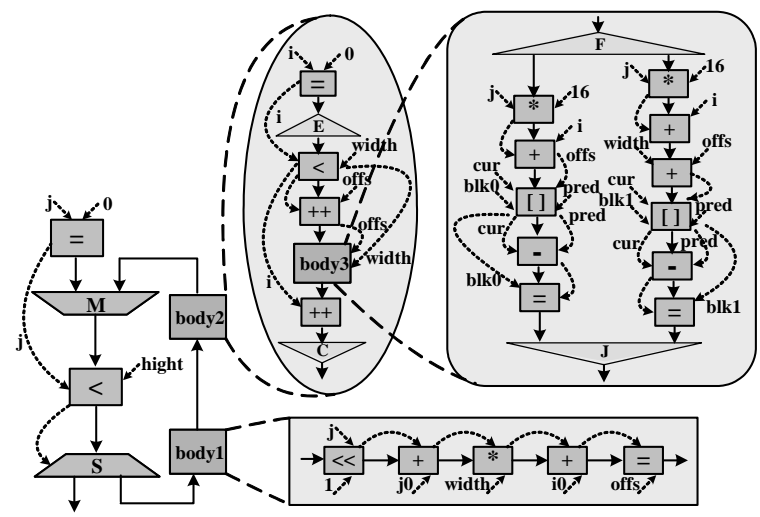

Fig. 8: HDG representation for the MPEG example.

Following the two guidelines mentioned above, we can find that the fourth statement is staggered from the fifth and sixth statements and they can be implemented with pipelining. Furthermore, the fifth and sixth statements also can be operated in parallel because there are no relations between them. The corresponding specification with HDG is shown in Figure 8. There are three hierarchical nodes and three special structures, pipelining, parallel and loop. If we adopt CDFG or other models, this character cannot be expressed. In this figure, the real lines stand for control flows and broken lines stand for data flows.

\section{Advantage compared with CDFG}

CDFG is a widely used model which has many same characters with HDG. For example, they are both composed with nodes and flows, and data dependency and control dependency can be represented both in these two representations.

However, there are two advantages in HDG compared with CDFG to supply necessary information for the instruction set customization:

(1) HDG has pipelining and parallel structures which support the assembly code optimization process. But this heterogeneous feature does not exist in CDFG. CDFG can only represent the nonparallel structures.
(2) For the generation of complex instruction set generation, the intermediate specification should be able to represent the hierarchical character. HDG solves this problem very well. As the example shown in section 5 , subset of nodes can be captured into a hierarchical node. However, CDFG can only represent the fine-granularity level graph.

From the compare above, we can find that HDG is a more appropriate choice than CDFG for representing the instruction set customization process.

\section{Conclusion}

In this paper, a novel intermediate representation named as HDG is presented to bridge the gap between application benchmarks and the process of instruction set customization. This specification is composed of nodes, flows and ports, which have four heterogeneous structures, pipelining, parallel, branch and loop. Those definitions ensure that it satisfies the requirements of instruction set customization.

Future work will focus on the automated conversion from the benchmarks written in high level languages to this intermediate representation.

\section{References}

[1] Manoj Kumar Jain, M. Balakrishnan, Anshul Kumar, “ASIP Design Methodologies: Survey and Issues”, Proceedings of the International Conference on VLSI Design, p.76, 2001.

[2] P. Biswas, V. Choudhary, K. Atasu, L. Pozzi, P. Ienne and N. Dutt, "Introduction of Local Memory Elements in Instruction Set Extensions”, Design Automation Conference (DAC '04), 2004.

[3] Peter Voigt Knudsen and Jan Madsen, “Graph Based Communication Analysis for HW/SW Codesign", Proceedings of the 7th International Workshop on Hardware/Software Codesign, Rome, Italy, May 3-5, 1999, pp. 131-135.

[4] Qiang Wu, Yunfeng Wang, Jinian Bian, Weimin Wu and Hongxi Xue, "A Hierarchical CDFG as Intermediate Representation for HW/SW Codesign”, Proceedings of the 2002 International Conference on Communications, Circuits and Systems, Chengdu, China, pp.1429-1432, 2002.

[5] http://poppy.snu.ac.kr/CDFG/cdfg.html, CDFG Toolkit, Seoul National University, Korea.

[6] SPARK 3-Layered Intermediate representation, http://mesl.ucsd.edu/spark/methodology/HTGs.s $\underline{\mathrm{html}}$

[7] Jonathan Alexander, "VHDL Design Tips and Low Power Design Techniques”, Applications Consulting Manager, Actel Corporation, 2004. 